\title{
Canonical and alternative transcript expression of PAX6 and CXCR4 in pancreatic cancer
}

\author{
ELIZABETH C. LITTLE ${ }^{1}$, JENNIFER D. KUBIC ${ }^{1}$, RAVI SALGIA $^{2}$, PAUL J. GRIPPO $^{3}$ and DEBORAH LANG ${ }^{1}$ \\ ${ }^{1}$ Department of Medicine, Section of Dermatology, University of Chicago, Chicago, IL 60637; \\ ${ }^{2}$ Department of Medical Oncology and Therapeutics Research, City of Hope, Duarte, CA 91010; \\ ${ }^{3}$ Department of Medicine, Division of Gastroenterology and Hepatology, \\ University of Illinois at Chicago, Chicago, IL 60612, USA
}

Received July 14, 2016; Accepted January 6, 2017

DOI: $10.3892 / 01.2017 .5956$

\begin{abstract}
Pancreatic cancer is a lethal disease with a propensity for invading and metastasizing into the surrounding tissues, including the liver and intestines. A number of factors are aberrantly overexpressed in this tumor type and actively promote cancer progression and metastasis. The present study demonstrates that paired box transcription factor 6 (PAX6) and $\mathrm{C}-\mathrm{X}-\mathrm{C}$ chemokine receptor 4 (CXCR4) are frequently co-expressed in primary pancreatic adenocarcinoma tumors and established cell lines. Expression analysis methods used in the present study included evaluation of protein expression by western blot analysis and immunofluorescence, transcript expression levels by reverse transcription-quantitative polymerase chain reaction (RT-qPCR), and luciferase assays utilizing regulatory elements from the CXCR4 gene locus. Canonical PAX6 and alternative splice variant PAX6(5a) proteins are expressed in pancreatic cancer and can drive gene expression through a conserved enhancer element within the first intron of the CXCR4 gene. As demonstrated by
\end{abstract}

Correspondence to: Dr Deborah Lang, Department of Medicine, Section of Dermatology, University of Chicago, 5841 S. Maryland Avenue, Mail Code 5067, Chicago, IL 60637, USA

E-mail: dlang@medicine.bsd.uchicago.edu

Dr Paul J. Grippo, Department of Medicine, Division of Gastroenterology and Hepatology, University of Illinois at Chicago, 840 South Wood Street, Room 738A, Chicago, IL 60612, USA E-mail: pgrippo@uic.edu

Abbreviations: CXCL12, C-X-C chemokine ligand 12; CXCR4, C-X-C chemokine receptor 4; CXCR4A, CXCR4 transcript variant $\mathrm{A}$; $\mathrm{CXCR} 4 \mathrm{~B}, \mathrm{CXCR} 4$ transcript variant $\mathrm{B}$; $\mathrm{kDa}$, kilodaltons; PAX6, paired box transcription factor 6; PAX6(5a), PAX6 exon 5 alternative transcript variant

Key words: pancreatic cancer, C-X-C chemokine receptor 4 , CXCR4 transcript variant $\mathrm{A}, \mathrm{CXCR} 4$ transcript variant $\mathrm{B}$, paired box transcription factor 6, PAX6 exon 5 alternative transcript variant, alternative splice variants the introduction of an exogenous reporter construct with or without the intronic enhancer, loss of this element inhibited gene expression within numerous pancreatic cancer cell lines including Panc1, MIA-PaCa2 and BxPC3. All of the pancreatic cancer cell lines expressed the canonical CXCR4B transcript in addition to the alternatively spliced variant CXCR4A as determined by RT-qPCR experiments. The discovery of variant transcripts in pancreatic cancer cells may provide new candidates for future targeted therapies.

\section{Introduction}

Pancreatic cancer is an aggressive type of cancer with an extremely poor prognosis (1). This type of tumor often metastasizes to multiple lymph nodes, with liver and peritoneal sites the most common for distant spread (2). The identification of molecular pathways and novel candidates for therapeutic targeting is urgently required to develop alternative treatments in order to improve clinical outcomes. Chemokine receptors have been implicated in pancreatic cancer metastasis (3). In particular, C-X-C chemokine receptor 4 (CXCR4), a chemokine G-protein-coupled receptor, is frequently expressed in pancreatic cancer and affects pancreatic tumor cell growth, adhesion, migration and invasion in cellulo and in clinical samples (4-8). The ligand for CXCR4, C-X-C chemokine ligand 12 (CXCL12; also known as stromal cell-derived factor 1 ) is expressed at high levels in tissues prone to pancreatic cancer spread, including the lymph nodes, liver and lung $(8,9)$. While the expression of CXCR4 is low or absent in the majority of healthy tissues, its expression is found in high frequency in pancreatic cancer specimens, ranging from $71.2-90 \%$ depending on the study $(4,6,8,9)$; however, the mechanism for CXCR4 misexpression is not well understood. A limited number of factors are associated with the regulation of CXCR4 expression in pancreatic cancer (10-12). For example, glycogen synthase kinase $3 \beta$ enhances CXCR4 expression (12), and CXCR4 expression is also promoted through the hypoxia-driven expression of the microRNA miRNA-150 (11). Additionally, the Boswellia serrata plant extract boswellic acid has been demonstrated to reduce CXCR4 levels in pancreatic cancer cells (10). As the CXCR4 receptor promotes pancreatic cancer growth, migration and metastasis, the targeting 
of factors that can downregulate CXCR4 has the potential to inhibit pancreatic cancer metastasis.

Our recent study revealed that paired box transcription factor (PAX3) regulates CXCR4 expression in melanoma (13). PAX6, a PAX3-associated protein, is expressed in pancreatic adenocarcinoma cell lines and tumor samples (14). PAX6 contains a paired-type DNA-binding domain that is similar to the paired domain of PAX3, and is able to bind to similar, but not identical, DNA sequences $(15,16)$. PAX6, like PAX3, interacts with the promoter of the receptor tyrosine kinase gene MET and promotes gene expression $(17,18)$. While PAX6 is normally expressed in the embryonic pancreatic bud and in pancreatic islet $\alpha$-cells, the expression of this factor is downregulated during exocrine pancreatic development and is absent in mature exocrine tissue (19). Studies investigating PAX6 function in pancreatic cancer have supported that PAX6 acts as a transcription factor (20); however, downstream effector genes of PAX6, other than MET (18), have not been reported.

PAX6 and CXCR4 have been previously identified to have alternative splice variants $(21,22)$. The canonical PAX6 protein has two DNA-binding domains (a bipartite paired domain and a paired-type homeodomain) and a $\mathrm{C}$-terminal transcriptional activation domain (23). A major splice variant of PAX6 is the exon 5 alternative transcript variant PAX6(5a), which possesses a 14-amino-acid insertion into an $\mathrm{N}$-terminal region of the paired domain which alters the DNA-binding preference (21). For the human CXCR4 gene, two major alternative splice variants have been identified: The canonical CXCR4B transcript, and CXCR4A (22). The two isoforms differ only in the furthest $\mathrm{N}$-terminal region of the protein, and this difference may affect how the receptor interacts with its major ligand, CXCL12 $(22,24)$. The expression and consequences of expression of these variant isoforms of PAX6 and CXCR4 in pancreatic cancer, and in cancer in general, are virtually unexplored.

The present study reports the expression of CXCR4 and PAX6 in pancreatic cancer primary tissues and cell lines, and indicates that there is a significant positive correlation of their co-expression. In parallel with our previous findings with PAX3, PAX6 is also sufficient to drive gene expression from the CXCR4 regulatory region, and this ability of PAX6 to promote transcription is dependent on the presence of a highly conserved intronic enhancer element. In addition, pancreatic cancer cell lines express the common transcripts of PAX6 and CXCR4, as well as the variant transcripts PAX6(5a) and CXCR4A. Canonical and/or variant forms of PAX6 and CXCR4 may participate in a shared molecular pathway in pancreatic cancer.

\section{Materials and methods}

Immunohistochemistry of primary tumor samples. A total of 22 primary tissue samples verified as late-stage pancreatic adenocarcinoma tumor specimens, obtained as autopsy specimens from the Cooperative Human Tissue Network, as previously described (25), were utilized in the current study; their use was compliant with and approved by the University of Chicago institutional review board and Clinical Trials Committees. Slides holding the tissue sections were boiled in
$1 \mathrm{X}$ citrate buffer for antigen retrieval. Samples were blocked in $1 \%$ normal goat serum, followed by overnight incubation at $4^{\circ} \mathrm{C}$ with primary antibodies against PAX6 (mouse monoclonal; dilution, 1:500; University of Iowa Hybridoma Bank, Iowa City, IA, USA) and CXCR4 (rabbit polyclonal; cat. no. Ab1670; dilution, 1:200; Abcam, Cambridge, MA, USA). Incubation for $1 \mathrm{~h}$ at $4^{\circ} \mathrm{C}$ with goat anti-rabbit fluorescein (cat. no. 31509; dilution, 1:1,000; Thermo Fisher Scientific, Inc., Waltham, MA, USA) or goat anti-mouse dyl647 (cat no. 21235; dilution, 1:1,000; Thermo Fisher Scientific, Inc) secondary antibodies, which enabled the fluorescence-mediated detection of antigens. Samples were scored as 'positive' or 'negative' for each antigen, with a positive score determined when $>25 \%$ of the tumor tissue expressed the antigen.

Cell culture and transfection. The pancreatic carcinoma cell lines AsPC-1, BxPC-3, Capan-2, HPAF II (HPAF), MIA PaCa-2, PANC-1, CFPAC-1 as well as control cells HEK-293T (human embryonic kidney) and 3T3 (fibroblast) were obtained from American Type Culture Collection (Manassas, VA, USA), the pancreatic carcinoma cell lines COLO-537, CD11 and SW979 were obtained from Dr Ruggeri from Allegeny University (Philadelphia, PA, USA) who was supplied them by Dr Batra from Eppley Institute for Research in Cancer (Omaha, NE, USA) (25), and all the cell lines were cultured as previously described $(14,18)$. Cells were transfected using Effectene Transfection Reagent (Qiagen, Inc., Valencia, CA, USA), according to manufacturer's protocols. In addition to the reporter and/or expression constructs, cells were also transfected with a $\beta$-galactosidase-expressing construct (pCMV; Clontech Laboratories, Inc., Mountain View, CA, USA) to serve as an internal control for luciferase assays. The total amount of DNA transfected per cell was maintained at a constant amount by the addition of pBluescript plasmid (Stratagene; Agilent Technologies, Santa Clara, CA, USA). Levels of luciferase and $\beta$-galactosidase were measured using assay kits from Promega (Promega Corporation, Madison, WI, USA) and each experiment was performed minimally in triplicate.

Vectors. The pGL2-CXCR4pm393, pGL2-CXCR4pm393L and pGL2-CXCR4pm393L- $\Delta$ ISH vectors were constructed as described previously (13). The pGL2-CXCR4pm393 construct contains a sequence of 393 bp 5'-proximal to exon 1 of the CXCR4 gene, as well as the 5' untranslated region (UTR) from exon 1. The pGL2-CXCR4pm393L vector contains the entire sequence of pGL2-CXCR4pm393 as well as the coding sequence from all of exon 1 and part of exon 2 cloned in-frame with the luciferase reporter gene cassette and the 1,781-bp intron 1. The pGL2-CXCR4pm393L- $\Delta$ ISH construct is the same as pGL2-CXCR4pm393L with the exception of a deletion of a 267-bp segment from intron 1 containing the island of homology with the PAX binding site.

Western blot analysis. Whole cell lysates from pancreatic cancer cells were isolated using RIPA buffer, loaded onto 4-15\% Tris-Bis gels (50 $\mu \mathrm{g}$ total protein per cell line) for electrophoresis, then transferred onto nitrocellulose membranes. Membranes were probed with an anti-PAX6 antibody and an anti- $\beta$-tubulin (cat no. E7) antibody (University of Iowa 
Hybridoma Bank; dilutions, 1:100 and 1:400, respectively). Immunoreactivity was detected using a WesternBreeze Chemiluminescent kit (cat. no. WB7104; Thermo Fisher Scientific, Inc.), according to the manufacturer's protocol. All incubations were performed at $4^{\circ} \mathrm{C}$ and for $1 \mathrm{~h}$ for primary and secondary antibody incubations and the blocking step. Secondary antibodies utilized were from the WesternBreeze kit. Following secondary antibody incubation the blots were washed at room temperature four times for five min for each wash. Densitometric analyses of resultant western blots were performed with ImageJ software (ImageJ version 1.47 public domain software; National Institutes of Health, Bethesda, MD, USA). For quantification, raw densitometry numbers were recorded for each band and normalized against the $\beta$-tubulin readings. The data presented are densitometric readings from three independent western blot analyses. Background readings are arbitrarily set at $0.5 \mathrm{U}$.

Reverse transcription-quantitative polymerase chain reaction $(R T-q P C R)$. RNA from pancreatic cancer cell lines was isolated using Trizol (Thermo Fisher Scientific, Inc.), DNase-treated with a Ambion DNA-free DNA Removal Kit (Thermo Fisher Scientific, Inc.), and used as a template for RT with the iScript cDNA Synthesis kit (Bio-Rad Laboratories, Inc., Hercules, CA, USA). The mRNA expression levels of CXCR4 transcript variants A and B (CXCR4A and CXCR4B, respectively), PAX6, PAX6(5a) and GAPDH were evaluated using SYBR-Green Master Mix (Bio-Rad Laboratories, Inc.) and the CFX Connect Real-Time System (Bio-Rad Laboratories, Inc.). The template for each RT-qPCR sample was $2.5 \mu \mathrm{l}$ of a 1:10 dilution of cDNA derived from $1 \mu \mathrm{g}$ of starting template RNA. Cycling conditions included 40 cycles of $95^{\circ} \mathrm{C}$ for $10 \mathrm{sec}$, annealing at $58^{\circ} \mathrm{C}$ for $10 \mathrm{sec}$ and a $30 \mathrm{sec} 72^{\circ} \mathrm{C}$ extension. The results are presented as the mean and standard error of the mean of three independent experiments, normalized against GAPDH expression and compared to the MIA PaCa- 2 cell line for relative expression using the Pfaffl method (26). MIA $\mathrm{PaCa}-2$ was chosen as the cell line of comparison due to this cell line having significant levels of all four transcripts. Primer sequences are presented in Table I.

Statistical analysis. GraphPad Prism statistical software (version 5.0; GraphPad Software, Inc., La Jolla, CA, USA) was utilized to determine whether the findings were significant. Correlation coefficients between PAX6-expressing and CXCR4-expressing tumor samples were determined with two-tailed Fisher's exact probability tests, with the analysis of nominal data as either positive or negative expression. The significance of the differences between the groups was determined with Student's t-test and $\chi^{2}$ analysis, with a confidence interval of $95 \%$. All values stated as significant have P-values of $\leq 0.05$, unless indicated. All experiments were performed in triplicate.

\section{Results}

PAX6 and CXCR4 are co-expressed in primary pancreatic adenocarcinoma samples. We have previously described PAX6 expression in pancreatic cancer established cell lines and primary tumor samples $(14,18)$. PAX6 is frequently
Table I. Primers utilized for the reverse transcription-quantitative polymerase chain reaction analysis.

Gene/primer name $\quad$ Sequence (5'-3')

\begin{tabular}{ll}
\hline CXCR4B forward & CCGAGGGCCTGAGTGCTCCAG \\
CXCR4A forward & GCAGAGGAGTTAGCCAAGATG \\
CXCR4 reverse & ATCCATTGCCCACAATGCCAG \\
PAX6 and PAX6(5a) & TTCAGAGCCCCATATTCGAG
\end{tabular}

forward

PAX6 reverse

PAX6(5a) reverse

\section{GTTGGACACCTGCAGAAT TGCATGGGTCTGCAGAAT}

CXCR4, C-X-C chemokine receptor 4; CXCR4B, CXCR4 transcript variant $\mathrm{B}$; CXCR4A, CXCR4 transcript variant A; PAX6, paired box transcription factor 6; PAX6(5a), PAX6 exon 5 alternative transcript variant.

co-expressed with the receptor protein CXCR4 (Fig. 1). In the present study, expression of PAX6 and CXCR4 was measured in 22 primary tumor tissues. Representative immunofluorescent staining results indicating the expression of PAX6 and CXCR4, as well as their co-expression, are shown in Fig. 1A. In the present study, 13/22 samples (59.1\%) and 15/22 samples (68.2\%) were positive for PAX6 and CXCR4 expression, respectively (Fig. 1B). The expression statuses of PAX6 and CXCR4 were positively correlated when compared using Fisher's exact probability tests $(\mathrm{P}=0.0066)$. Of the PAX6-expressing tumor samples, the majority $(12 / 13 ; 92.3 \%)$ also co-expressed CXCR4.

PAX6 activates a reporter containing CXCR4 gene elements. We previously demonstrated that the PAX transcription factor PAX3 promotes CXCR4 expression in melanoma through a highly conserved enhancer in the CXCR4 gene (13). The PAX enhancer element is located within the first intron of the CXCR4 gene, immediately 3'-proximal to exon 1 (Fig. 2A). The element (Fig. 2A, black box) is located in a 52-bp sequence that is highly conserved between mammals [Fig. 2A, grey box (13)]. PAX3 was found to bind to this PAX element. The PAX6 paired domain binds to a sequence with a TT(A/C) ACGC(A/T) core, first identified through in vitro site selection assays (15) and recently supported through in cellulo chromatin immunoprecipitation-sequencing and systematic evolution of ligands by exponential enrichment studies $(27,28)$. The PAX site in the CXCR4 enhancer region closely matches the preferred PAX6 site, and is a more ideal site for PAX6 over PAX3 due to the 5 ' TT rather than GT sequence $(15,16)$. To examine whether PAX6 is also capable of driving expression from this element, vectors containing regions of the CXCR4 locus were transfected into 293T cells with or without PAX6 and/or PAX6(5a), an alternative splice version of PAX6. Three constructs were tested: pGL2-CXCR4pm393, pGL2-CXCR4pm393L and pGL2-CXCR4pm393L- $\Delta$ ISH. The pGL2-CXCR4pm393 vector contains 393 bp of sequence from 5'-proximal to exon 1 and the 5'-UTR. The pGL2-CXCR4pm393L vector contains all the sequence of pGL2-CXCR4pm with the addition of the first intron and part of the coding sequence cloned in-frame with 
A

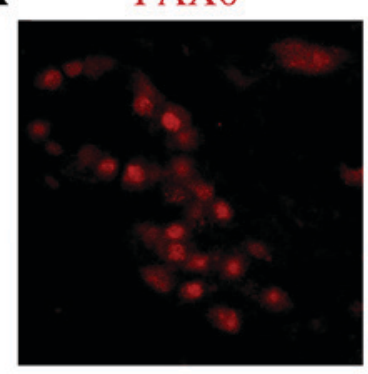

CXCR4

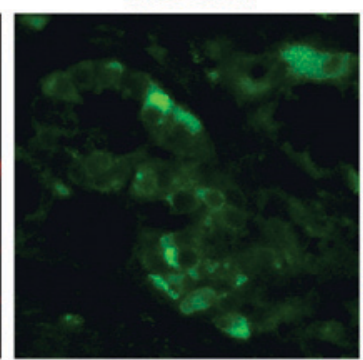

Merge

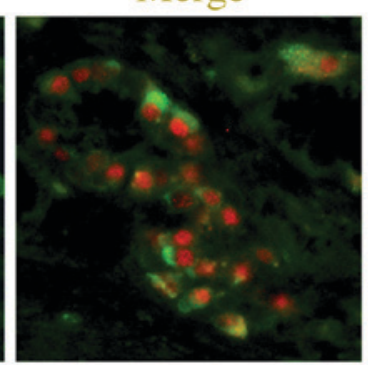

DAPI

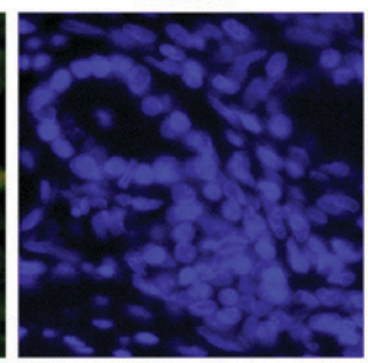

B

\begin{tabular}{|r|c|c|c|}
\hline & PAX6+ & PAX6- & Total \\
\hline CXCR4+ & 12 & 3 & 15 \\
\hline CXCR4- & 1 & 6 & 7 \\
\hline Total & 13 & 9 & 22 \\
\hline
\end{tabular}

Figure 1. PAX6 and CXCR4 are expressed in primary human pancreatic cancer tissue. (A) Representative pancreatic cancer specimen with immunofluorescence staining for PAX6 (red) and CXCR4 (green), as well as the merged images of the two antigens and the corresponding DAPI staining (blue) to indicate cell nuclei. The sample shown had a ductal lesion with desmoplastic fibroblasts around the tumor cells. (B) A summary table of a positive (+) and negative (-) expression patterns for PAX6 and CXCR4 in pancreatic adenocarcinoma tumor samples. PAX6, paired box transcription factor 6; CXCR4, C-X-C chemokine receptor 4.

the luciferase gene cassette. The pGL2-CXCR4pm393L- $\Delta$ ISH vector has the same elements as pGL2-CXCR4pm393L, but with a deletion of the island of sequence homology. These vectors are shown schematically in Fig. 2B. PAX6 or PAX6(5a) did not drive reporter expression from the pGL2-CXCR4pm393 vector (Fig. 2C). However, PAX6 and PAX6(5a) induced significant reporter expression from the pGL2-CXCR4pm393L construct containing an intact intron $1[2.8 \pm 0.69$-fold $(\mathrm{P}=0.011)$ and $3.8 \pm 0.63$-fold $(\mathrm{P}=0.0017)$ for PAX6 and PAX6(5a), respectively, relative to the reporter vector alone]. The combined addition of PAX6 and PAX6(5a) produced the highest level of reporter expression (8.1 \pm 0.25 -fold). Deletion of the enhancer element within the intron completely eliminated the ability of either of the PAX6 protein variants to drive luciferase expression.

In order to determine whether the enhancer site containing the PAX element drives expression in pancreatic cancer cells, the three vectors were transfected into PANC-1, MIA PaCa-2, and BxPC-3 cell lines. All cell lines exhibited luciferase activity from the pGL2-CXCR4pm393L vector (set at 100\%), and activity was significantly decreased when the intronic enhancer element was removed (pGL2-CXCR4pm393L- $\triangle \mathrm{ISH}$ ): $57.09 \pm 17.80 \%$ in PANC-1 cells $(\mathrm{P}=0.035), 47.7 \pm 23.11 \%$ in $\mathrm{MIA} \mathrm{PaCa}-2$ cells $(\mathrm{P}=0.034)$, and $42.39 \pm 19.18 \%$ in $\mathrm{BxPC}-3$ cells $(\mathrm{P}=0.026$; Fig. 2D). The expression of luciferase reporter was significantly lower compared with pGL2-CXCR4pm393L, when only the proximal promoter vector was utilized (pGL2-CXCR4pm393) in all cell lines: PANC-1, 16.32 $\pm 8.48 \%(\mathrm{P}=0.0025)$; MIA $\mathrm{PaCa}-2,11.82 \pm 5.25 \%(\mathrm{P}=0.0031)$; and $\mathrm{BxPC}-3,15.53 \pm 11.74 \%$ $(\mathrm{P}=0.0059)$. These data support the hypothesis that PAX6 proteins drive the CXCR4 gene through a conserved intronic element, and that the loss of this site leads to a significant loss of enhancer activity in pancreatic cancer cells.

Pancreatic cancer cell lines express PAX6. We have previously demonstrated that PAX6 is expressed in pancreatic carcinoma primary tumors and cell lines (14). Furthermore, unexpectedly, the predominant PAX6 protein expressed in SW979, PANC-1, and MIA PaCa-2 cells was found to be the alternatively spliced 48-kDa version, PAX6(5a), rather than the canonical $46 \mathrm{kDa}$ protein (18). In the present study, PAX6 levels were evaluated in nine independent pancreatic cell lines (Fig. 3A and B). All the cell lines expressed measurable levels of PAX6 protein, except for the low or undetectable levels in HPAF cells. As demonstrated in our aforementioned results, the predominant band was indicated to be $\sim 48 \mathrm{kDa}$, corresponding to the alternative rather than canonical form of PAX6 (Fig. 3C). Bands at $46 \mathrm{kDa}$ were absent or faint. To determine PAX6 transcript levels, RT-qPCR analysis utilizing transcript-specific primers were performed. The PAX6(5a) transcript was expressed in all eight of the cell lines tested, while 5/8 lines produced the canonical transcript (Fig. 3D); the cell lines AsPC-1, COLO-357, and SW979 did not have measureable levels of PAX6 canonical transcript. No correlation was identified between the levels of transcript and the detected levels of protein. However, HPAF cells expressed significant levels of the two transcripts while producing low or no PAX6 protein.

Pancreatic cancer cell lines express variable levels of CXCR4A and CXCR4B transcripts. CXCR4 protein is widely expressed in pancreatic cancer, with significant levels detected in various pancreatic cancer cell lines, including all lines used in the present study $(4-6,29,30)$. Previous reports have identified CXCR4 expression in pancreatic cell lines by RT-PCR, western blot analysis and immunohistochemistry; however, these methods do not differentiate between the two major versions of CXCR4 (canonical CXCR4 protein/CXCR4B transcript and CXCR4-Lo protein/CXCR4A transcript). The human CXCR4 gene comprises two exons and two alternative start codons (Fig. 4A) (22). The two alternatively spliced 


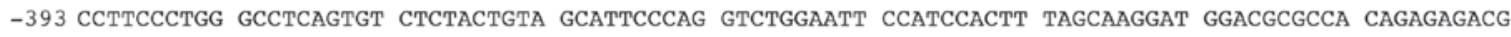

-303 CGTTCCTAGC CCGCGCTTCC CACCTGTCTT CAgGCGCATC CCGCTTCCCT CAAACTTAgG AAATGCCTCT GGgAgGTCCT GTCCGGCTCC

-213 GGACTCACTA CCGACCACCC GCAAACAGCA GGGTCCCCTG GGCTTCCCAA GCCGCGCACC TCTCCGCCCC GCCCCTGCGC CCTCCTTCCT -123 CGCGTCTGCC сCтCTCCCCC ACCCCGCCTT CTCCCTCCCC GCCCCAGCGG CGCATGCGCC GCGCTCGGAG CGTGTTTTTA TAAAATACCG -33 GCCGCGGCCA GAAACTTCAG TTTGTTGgCT GCGGCAGCAG GTAGCAAAgT GACGCCGAGg GCCTGAGTGC TCCAGTAGCC ACCGCATCTG +57 GAGAACCAGC GGTTACCATG GAGGGATCA GT GTAAGTCC AGTTTCAACC TGCTTTGTCA TAAATGTACA AACGTTTGAA CTTAGAGCGC

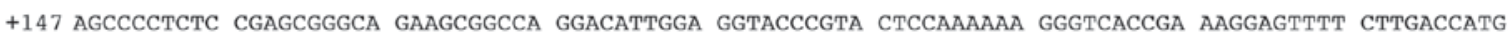

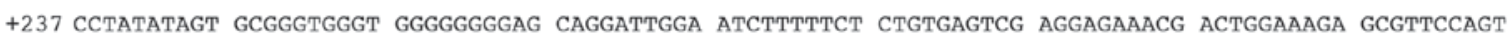

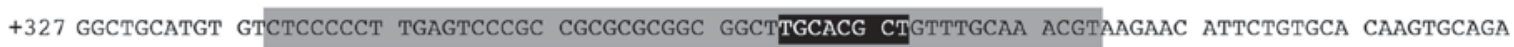

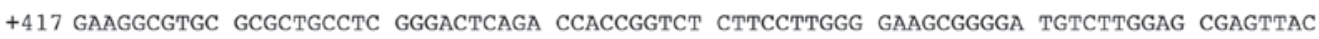

B

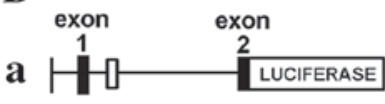

b

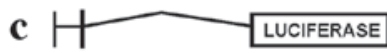

C

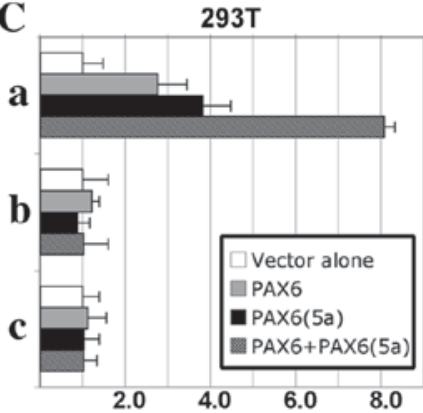

Reporter expression induction (fold)

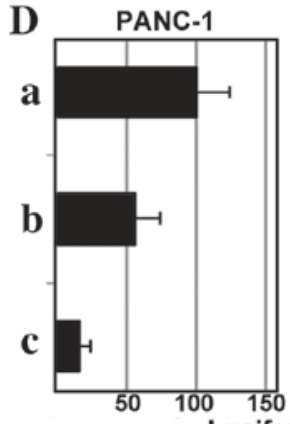

MIA PaCa-2

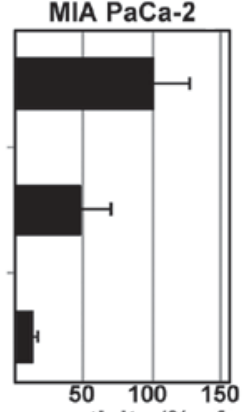

Luciferase activity $(\%$ of vector a)
BxPC3

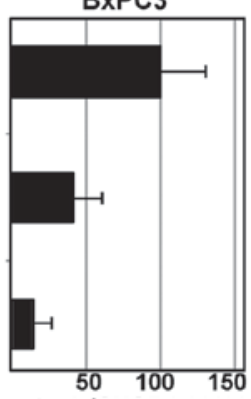

Figure 2. The CXCR4 locus contains a conserved enhancer element within intron 1 that is PAX6-responsive and active in pancreatic cancer cells. (A) Sequence of a section of the human CXCR4 locus, containing 393 bp 5'-proximal to exon 1, exon 1 (box) with the translational start codon (ATG in bold), and partial intron 1 sequence. The intronic sequence shown contains a 52-bp island of high conservation of homology between mammals (grey highlight), as well as a previously identified PAX site (black highlight, white letters) (13) within this homologous region. (B) Schematic of CXCR4 gene expression constructs: (a) pGL2-CXCR4pm393L vector containing 393 bp of the sequence 5'-proximal to exon 1 of the CXCR4 gene, the 5'-untranslated region from exon 1 and the coding sequence from all of exon 1 and part of exon 2, and the 1,781-bp intron 1; (b) pGL2-CXCR4pm393L- $\triangle$ ISH construct is the same as pGL2-CXCR4pm393L except for a deletion of a 267-bp segment from intron 1, containing the island of homology shown in the grey shaded region in (A); (c) pGL2-CXCR4pm393 construct contains only the 393-bp sequence 5'-proximal to exon 1, and none of the other regions present in the pGL2-CXCR4pm393L vector. (C) PAX6 proteins promote gene expression through a highly conserved enhancer in the CXCR4 intron. The different reporter constructs (a, pGL2-CXCR4pm393L; b, pGL2-CXCR4pm393L- $\mathrm{ISH}$; or c, pGL2-CXCR4pm393) were transfected into 293T cells with a reporter vector alone or with expression constructs expressing canonical PAX6, PAX6(5a), or both PAX6 and PAX6(5a) isoforms. Luciferase induction is shown as the fold difference relative to the reporter vector alone, which was set at 1-fold. (D) Loss of the conserved intronic enhancer element from the CXCR4 gene diminishes the activity of CXCR4 reporter constructs in pancreatic cancer cell lines. Reporter vectors (a, pGL2-CXCR4pm393L; b, pGL2-CXCR4pm393L- $I$ ISH; or c, pGL2-CXCR4pm393) were transfected into PANC-1, MIA PaCa-2 and BxPC-3 cells. Light levels measured following transfection with vector a (pGL2-CXCR4pm393L) were set at 100\% . CXCR4, C-X-C chemokine receptor 4; PAX6, paired box transcription factor 6; PAX6(5a), PAX6 exon 5 alternative transcript variant.

transcripts (CXCR4B and CXCR4A) differ from one another at the N-terminal ends. CXCR4B (also known as CXCR4 variant 2) encodes the more common CXCR4 protein utilizing codons from exons 1 and 2, while CXCR4A (also referred to as CXCR4 variant 1) produces the longer CXCR4-Lo protein and is encoded entirely from exon 2 (Fig. 4B). The canonical protein encoded from the CXCR4B transcript is expressed in a wide array of tissues, while CXCR4-Lo expression is normally restricted to peripheral blood lymphocytes and spleen cells (22). The proteins differ only in the furthest six or nine (CXCR4 or CXCR4-Lo, respectively) amino acids of the N-terminal ends and, although the functional consequence of this difference is unknown, there is evidence of a differential response to ligand binding $(22,24)$. Our previous study revealed that the canonical CXCR4B transcript was the dominant transcript in melanoma, with expression in all seven lines analyzed (13). However, there was measurable expression of the CXCR4A transcript in three of the seven melanoma lines, albeit to significantly lower levels than those of the canonical transcript. In the present study, pancreatic cancer cell lines were found to express measurable levels of the CXCR4A and CXCR4B transcripts in all eight cell lines measured (Fig. 4C).

\section{Discussion}

The present study identified that, while both of the PAX6 transcripts are expressed in pancreatic cancer cell lines, the PAX6(5a) variant form is expressed in all eight cell lines tested and produces the majority of the PAX6 protein (Fig. 3). It is not known why PAX6 is aberrantly expressed in pancreatic cancer, or why the PAX6(5a) variant is the predominant form expressed. The two proteins are identical with the exception of a 14-amino-acid insertion into the N-terminal of the paired DNA-binding domain (22). This insertion alters the DNA-binding specificity of the domain, inducing the two PAX6 proteins to have various DNA binding site preferences and affinities. The PAX6 and PAX6(5a) paired domains bind to associated but distinct core DNA elements, with lower affinities for the preferred DNA binding site of the 
A

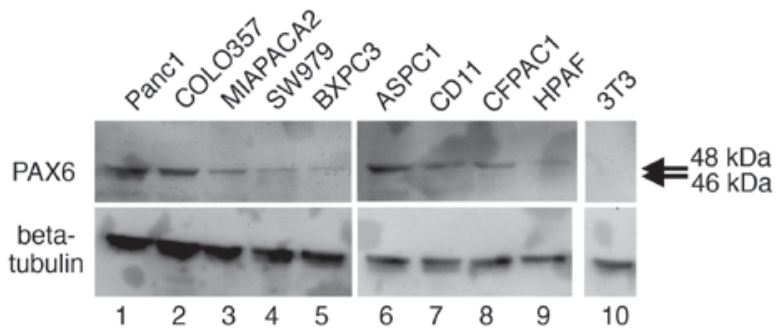

B

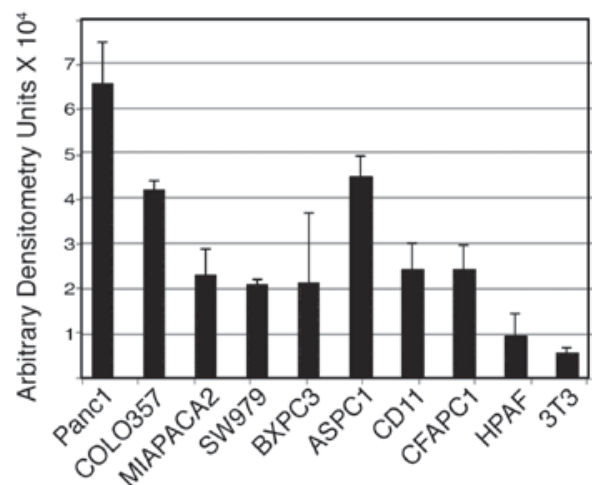

C

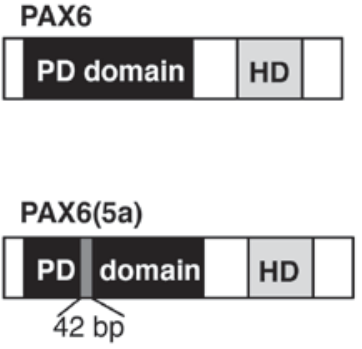

D

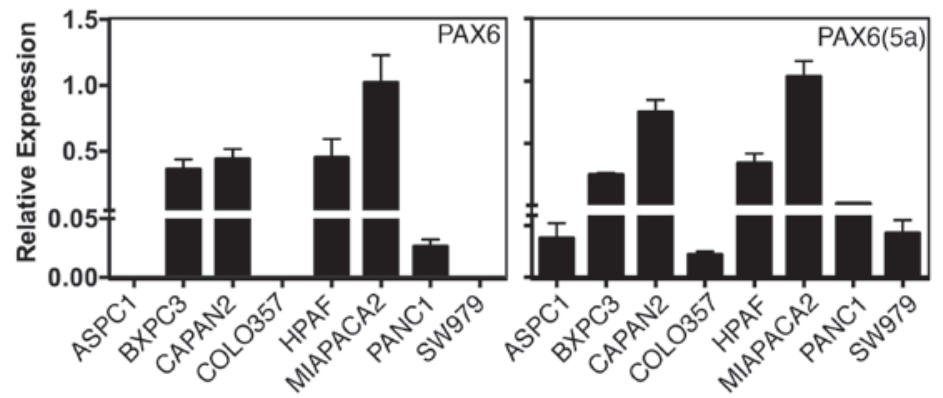

Figure 3. Pancreatic cancer cell lines express PAX6. (A) Protein expression of PAX6 in pancreatic cancer cell lines. PAX6 is expressed at variable levels in a panel of cell lines (lanes 1-9) but not in mouse fibroblast 3 T3 cells (lane 10). The protein size is predominantly $48 \mathrm{kDa}$ [as determined previously (18)], which corresponds to the PAX6 variant protein PAX6(5a). (B) Densitometry reading of three independent western blot analyses. Background readings are arbitrarily set at 0.5 units. (C) Schematic of canonical PAX6 and PAX6(5a) proteins. The proteins are identical except for an inserted 42-bp segment in exon 5, leading to an additional 14 amino acids in PAX6(5a). (D) Reverse transcription quantitative polymerase chain reaction analysis of PAX6 and PAX6(5a) transcripts in a panel of pancreatic cancer cell lines. Bars represent three independent experiments. Relative expression units are set so that a unit of 1 equals the average total levels of transcript in MIA PaCa-2 cells. PAX6, paired box transcription factor 6; PAX6(5a), PAX6 exon 5 alternative transcript variant; PD domain, paired-type DNA-binding domain; HD, homeodomain.

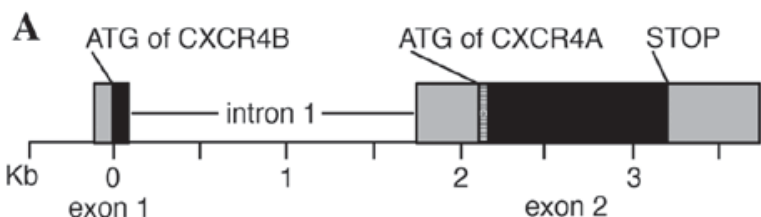

C

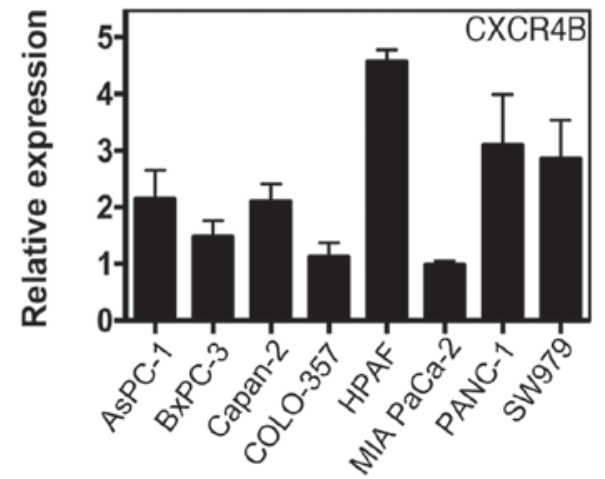

B

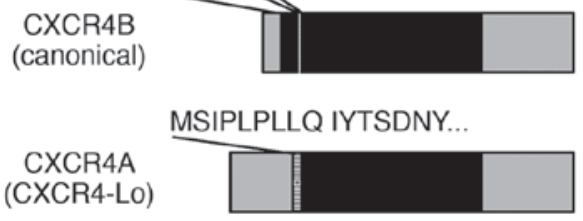

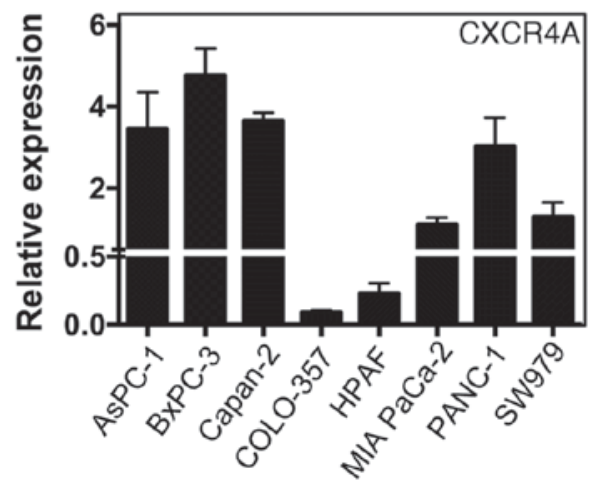

Figure 4. Pancreatic cancer cell lines express variable levels of CXCR4A and CXCR4B transcripts. (A) Schematics of the CXCR4 gene locus, including exon 1 with the start site for CXCR4B (canonical transcript), intron 1, alternative start site for CXCR4A (alternative transcript CXCR4-Lo), and exon 2, including the stop codon and the $3^{\prime}$ untranslated region. (B) Schematics for the CXCR4B transcript encoding the canonical CXCR4 protein, and the alternatively spliced CXCR4A transcript of the CXCR4-Lo protein. The resulting protein products are identical except for short peptides at the N-terminal ends. (C) Reverse transcription quantitative polymerase chain reaction analysis of CXCR4A and CXCR4B transcripts in a panel of pancreatic cancer cell lines. Bars represent three independent experiments. Relative expression units are set so that a unit of 1 equals the average total levels of transcript in MIA PaCa-2 cells. CXCR4, C-X-C chemokine receptor 4; CXCR4A, CXCR4 transcript variant A; CXCR4B, CXCR4 transcript variant B. 
other protein $(15,21,31)$. In the present study, no difference in gene expression between PAX6 and PAX6(5a) proteins was measured in CXCR4 gene reporter assays (Fig. 2C). In a previous study, expressing PAX6 or PAX6(5a) in a cell line without endogenous PAX6 expression (mouse fibroblast 3T3 cells) identified genes that were regulated by either of the PAX6 proteins or were subject to transcript-specific gene regulation (32). In addition, isoform-specific PAX6 mouse mutants have overlapping but different phenotypes $(33,34)$, and this is in parallel with human studies wherein a specific PAX6(5a) mutation has been shown to have similar abnormalities but does not phenocopy other PAX6 mutations (35). These findings support the hypothesis that PAX6 proteins have redundant and unique functions.

While canonical PAX6 was found to be expressed in five of the eight pancreatic cancer cell lines (Fig. 3D), the major PAX6 product expressed was the larger PAX6(5a) protein [Fig. 3A and our previous study (18)]. In certain normal tissues and during development, the two isoforms are expressed together and can functionally interact (36). The transcriptional function of the two isoforms is influenced by the ratio of PAX6:PAX6(5a); depending on cell type and developmental stage, the optimal balance ranges from 8:1-3:1 in the brain (depending on developmental stage) to $1: 1$ in the developing and maturing retina (31,37-39). In the present study, the two variants promoted the expression of a reporter gene containing CXCR4 gene elements (Fig. 2B). While the expression of the reporter was significantly increased with the addition of both isoforms, it is not clear whether expression would be altered or optimized utilizing the protein ratios found in pancreatic cancer cells. Notably, in these cancer cells, the majority of the protein is the variant form, which is the opposite of what is found in normal tissues wherein the canonical is the dominant form. What remains unknown is how the interaction of the two isoforms affects gene expression, whether this is purely due to DNA site binding specificity or due to changes in interactions with protein co-factors, and how this impacts pancreatic cancer selection, survival or progression.

This present study provides evidence that PAX6 is in a common molecular pathway with CXCR4, since the two proteins are expressed together (Fig. 1), and that PAX6 proteins are sufficient to drive expression through an intronic element within the CXCR4 gene (Fig. 2). Loss of this conserved intronic enhancer led to a decrease in gene expression in three pancreatic cancer cell lines (Fig. 2D). It is not clear whether inhibition of one or both of the PAX6 isoforms would lead to alteration of CXCR4 expression in pancreatic cancer, or if PAX6 drives expression of CXCR4A and CXCR4B equally or if there is specificity. The present results identified the expression of the canonical CXCR4B transcript as well as the CXCR4A variant (Fig. 4). While expression of the CXCR4B transcript is well documented in many types of human cancer, the presence of the alternative CXCR4A variant is not well studied. Indeed, the expression of CXCR4A even in normal mature tissues is highly restricted to peripheral blood lymphocytes and spleen cells (22). In cancer cells, our group identified expression in a subset of melanoma cells (13). In addition, the two transcripts are expressed in Ewing sarcoma primary tissue and cell lines (40). It is not clear how common the expression of CXCR4A transcript is in cancer in general, and what is the potential impact of its expression. However, since the extracellular domain differs between the receptors, this variance may be exploited in the development of targeted therapies specific for the more cell-type restricted CXCR4A.

In conclusion, the present study revealed that PAX6 and CXCR4 are co-expressed in pancreatic cancer, may be part of a shared pathway, and are each expressed as their respective canonical and variant transcripts. These present findings suggest that PAX6 and CXCR4 are good candidates for therapies, and the presence of the non-canonical proteins may provide novel targets for future therapeutics.

\section{Acknowledgements}

This study was supported in whole or in part by the National Institutes of Health (grant nos. NIH R01CA130202, NIH R01CA184001 and NIH P30-CA014599), the American Cancer Society (grant no. RSG-CSM-121505) and the Friends of Dermatology-Chicago.

\section{References}

1. Ilic $\mathrm{M}$ and Ilic I: Epidemiology of pancreatic cancer. World $\mathrm{J}$ Gastroenterol 22: 9694-9705, 2016.

2. Wolfgang CL, Herman JM, Laheru DA, Klein AP, Erdek MA, Fishman EK and Hruban RH: Recent progress in pancreatic cancer. CA Cancer J Clin 63: 318-348, 2013.

3. Marchesi F, Grizzi F, Laghi L, Mantovani A and Allavena P: Molecular mechanisms of pancreatic cancer dissemination: The role of the chemokine system. Curr Pharm Des 18: 2432-2438, 2012.

4. Koshiba T, Hosotani R, Miyamoto Y, Ida J, Tsuji S, Nakajima S, Kawaguchi M, Kobayashi H, Doi R, Hori T, et al: Expression of stromal cell-derived factor 1 and CXCR4 ligand receptor system in pancreatic cancer: A possible role for tumor progression. Clin Cancer Res 6: 3530-3535, 2000.

5. Mori T, Doi R, Koizumi M, Toyoda E, Ito D, Kami K, Masui T, Fujimoto K, Tamamura H, Hiramatsu K, et al: CXCR4 antagonist inhibits stromal cell-derived factor 1-induced migration and invasion of human pancreatic cancer. Mol Cancer Ther 3: 29-37, 2004.

6. Wehler T, Wolfert F, Schimanski CC, Gockel I, Herr W, Biesterfeld S, Seifert JK, Adwan H, Berger MR, Junginger T, et al: Strong expression of chemokine receptor CXCR4 by pancreatic cancer correlates with advanced disease. Oncol Rep 16: 1159-1164, 2006.

7. Marchesi F, Monti P, Leone BE, Zerbi A, Vecchi A, Piemonti L, Mantovani A and Allavena P: Increased survival, proliferation, and migration in metastatic human pancreatic tumor cells expressing functional CXCR4. Cancer Res 64: 8420-8427, 2004.

8. Gebauer F, Tachezy M, Effenberger K, von Loga K, Zander H, Marx A, Kaifi JT, Sauter G, Izbicki JR and Bockhorn M: Prognostic impact of CXCR4 and CXCR7 expression in pancreatic adenocarcinoma. J Surg Oncol 104: 140-145, 2011.

9. Marechal R, Demetter P, Nagy N, Berton A, Decaestecker C, Polus M, Closset J, Deviére J, Salmon I and Van Laethem JL: High expression of CXCR4 may predict poor survival in resected pancreatic adenocarcinoma. Br J Cancer 100: 1444-1451, 2009.

10. Park B, Sung B, Yadav VR, Cho SG, Liu M and Aggarwal BB: Acetyl-11-keto- $\beta$-boswellic acid suppresses invasion of pancreatic cancer cells through the downregulation of CXCR4 chemokine receptor expression. Int J Cancer 129: 23-33, 2011.

11. Sun JS, Zhang XL, Yang YJ, Nie ZG and Zhang Y: Hypoxia promotes $\mathrm{C}-\mathrm{X}-\mathrm{C}$ chemokine receptor type 4 expression through microRNA-150 in pancreatic cancer cells. Oncol Lett 10: 835-840, 2015.

12. Ying X, Jing L, Ma S, Li Q, Luo X, Pan Z, Feng Y and Feng P: GSK3 $\beta$ mediates pancreatic cancer cell invasion in vitro via the CXCR4/MMP-2 Pathway. Cancer Cell Int 15: 70, 2015.

13. Kubic JD, Lui JW, Little EC, Ludvik AE, Konda S, Salgia R, Aplin AE and Lang D: PAX3 and FOXD3 promote CXCR4 expression in melanoma. J Biol Chem 290: 21901-21914, 2015. 
14. Lang D, Mascarenhas JB, Powell SK, Halegoua J, Nelson M and Ruggeri BA: PAX6 is expressed in pancreatic adenocarcinoma and is downregulated during induction of terminal differentiation. Mol Carcinog 47: 148-156, 2008.

15. Epstein J, Cai J, Glaser T, Jepeal L and Maas R: Identification of a Pax paired domain recognition sequence and evidence for DNA-dependent conformational changes. J Biol Chem 269: 8355-8361, 1994

16. Goulding MD, Chalepakis G, Deutsch U, Erselius JR and Gruss P: Pax-3, a novel murine DNA binding protein expressed during early neurogenesis. Embo J 10: 1135-1147, 1991.

17. Mascarenhas JB, Littlejohn EL, Wolsky RJ, Young KP, Nelson M, Salgia R and Lang D: PAX3 and SOX10 activate MET receptor expression in melanoma. Pigment Cell Melanoma Res 23: 225-237, 2010.

18. Mascarenhas JB, Young KP, Littlejohn EL, Yoo BK, Salgia R and Lang D: PAX6 is expressed in pancreatic cancer and actively participates in cancer progression through activation of the MET tyrosine kinase receptor gene. J Biol Chem 284: 27524-27532, 2009.

19. St-Onge L, Sosa-Pineda B, Chowdhury K, Mansouri A and Gruss P: Pax6 is required for differentiation of glucagon-producing alpha-cells in mouse pancreas. Nature 387: 406-409, 1997.

20. Epstein JA, Lam P, Jepeal L, Maas RL and Shapiro DN: Pax3 inhibits myogenic differentiation of cultured myoblast cells. J Biol Chem 270: 11719-11722, 1995.

21. Epstein JA, Glaser T, Cai J, Jepeal L, Walton DS and Maas RL: Two independent and interactive DNA binding subdomains of the PAX6 paired domain are regulated by alternative splicing. Genes Dev 8: 2022-2034, 1994.

22. Gupta SK and Pillarisetti K: Cutting edge: CXCR4-Lo: Molecular cloning and functional expression of a novel human CXCR4 splice variant. J Immunol 163: 2368-2372, 1999.

23. Glaser T, Walton DS and Maas RL: Genomic structure, evolutionary conservation and aniridia mutations in the human PAX6 gene. Nat Genet 2: 232-239, 1992.

24. Duquenne C, Psomas C, Gimenez S, Guigues A, Carles MJ, Barbuat C, Lavigne JP, Sotto A, Reynes J, Guglielmi P, et al: The two human CXCR4 isoforms display different HIV receptor activities: Consequences for the emergence of X4 strains. J Immunol 193: 4188-4194, 2014.

25. Ruggeri BA, Huang L, Berger D, Chang H, Klein-Szanto AJ, Goodrow T, Wood M, Obara T, Heath $\mathrm{CW}$ and Lynch $\mathrm{H}$ : Molecular pathology of primary and metastatic ductal pancreatic lesions: Analyses of mutations and expression of the p53, mdm-2 and p21/WAF-1 genes in sporadic and familial lesions. Cancer 79: 700-716, 1997.

26. Pfaffl MW: A new mathematical model for relative quantification in real-time RT-PCR. Nucleic Acids Res 29: e45, 2001.

27. Sun J, Rockowitz S, Xie Q, Ashery-Padan R, Zheng D and Cvekl A: Identification of in vivo DNA-binding mechanisms of Pax6 and reconstruction of Pax6-dependent gene regulatory networks during forebrain and lens development. Nucleic Acids Res 43: 6827-6846, 2015
28. Bryne JC, Valen E, Tang MH, Marstrand T, Winther O, da Piedade I, Krogh A, Lenhard B and Sandelin A: JASPAR, the open access database of transcription factor-binding profiles: New content and tools in the 2008 update. Nucleic Acids Res 36 (Database Issue): D102-D106, 2008.

29. Heinrich EL, Lee W, Lu J, Lowy AM and Kim J: Chemokine CXCL12 activates dual CXCR4 and CXCR7-mediated signaling pathways in pancreatic cancer cells. J Transl Med 10: 68, 2012.

30. Singh S, Srivastava SK, Bhardwaj A, Owen LB and Singh AP: CXCL12-CXCR4 signalling axis confers gemcitabine resistance to pancreatic cancer cells: A novel target for therapy. Br J Cancer 103: 1671-1679, 2010.

31. Kozmik Z, Czerny T and Busslinger M: Alternatively spliced insertions in the paired domain restrict the DNA sequence specificity of Pax6 and Pax8. Embo J 16: 6793-6803, 1997.

32. Kiselev Y, Eriksen TE, Forsdahl S, Nguyen LH and Mikkola I: 3 T3 cell lines stably expressing Pax6 or Pax6(5a)-a new tool used for identification of common and isoform specific target genes. PLoS One 7: e31915, 2012.

33. Duncan MK, Kozmik Z, Cveklova K, Piatigorsky J and Cvekl A: Overexpression of PAX6(5a) in lens fiber cells results in cataract and upregulation of (alpha)5(beta)1 integrin expression. J Cell Sci 113: 3173-3185, 2000.

34. Singh S, Mishra R, Arango NA, Deng JM, Behringer RR and Saunders GF: Iris hypoplasia in mice that lack the alternatively spliced Pax6(5a) isoform. Proc Natl Acad Sci USA 99: 6812-6815, 2002.

35. Azuma N, Yamaguchi Y, Handa H, Hayakawa M, Kanai A and Yamada M: Missense mutation in the alternative splice region of the PAX6 gene in eye anomalies. Am J Hum Genet 65: 656-663, 1999.

36. Chauhan BK, Reed NA, Zhang W, Duncan MK, Kilimann MW and Cvekl A: Identification of genes downstream of Pax6 in the mouse lens using cDNA microarrays. J Biol Chem 277: 11539-11548, 2002.

37. Chauhan BK, Yang Y, Cveklová K and Cvekl A: Functional interactions between alternatively spliced forms of Pax6 in crystallin gene regulation and in haploinsufficiency. Nucleic Acids Res 32: 1696-1709, 2004.

38. Pinson J, Mason JO, Simpson TI and Price DJ: Regulation of the Pax6: Pax6(5a) mRNA ratio in the developing mammalian brain. BMC Dev Biol 5: 13, 2005.

39. Zhang W, Cveklova K, Oppermann B, Kantorow M and Cvekl A: Quantitation of PAX6 and PAX6(5a) transcript levels in adult human lens, cornea, and monkey retina. Mol Vis 7: 1-5, 2001.

40. Sand LG, Scotlandi K, Berghuis D, Snaar-Jagalska BE, Picci P, Schmidt T, Szuhai K and Hogendoorn PC: CXCL14, CXCR7 expression and CXCR4 splice variant ratio associate with survival and metastases in Ewing sarcoma patients. Eur J Cancer 51: 2624-2633, 2015. 